\title{
Interplay of Neutrino Flavor, Spin and Collective Oscillations in Supernovae
}

\author{
Konstantin Kouzakov, ${ }^{a}$ Yu-Feng Li, ${ }^{b, c, *}$ Vadim Shakhov, ${ }^{a}$ Konstantin Stankevich, ${ }^{a}$ \\ Alexander I. Studenikin ${ }^{a, d}$ and Ziyi Yuan ${ }^{b, e}$ \\ ${ }^{a}$ Faculty of Physics, Lomonosov Moscow State University, Moscow 119991, Russia \\ ${ }^{b}$ Institute of High Energy Physics, Chinese Academy of Sciences, Beijing 100049, China \\ ${ }^{c}$ School of Physical Sciences, University of Chinese Academy of Sciences, Beijing 100049, China \\ ${ }^{d}$ Joint Institute for Nuclear Research, Dubna 141980, Moscow Region, Russia \\ ${ }^{e}$ School of Physics and Technology, Wuhan University, Wuhan 430072, China \\ E-mail: liyufeng@ihep.ac.cn
}

Future large neutrino detectors, such as JUNO, Hyper-Kamiokande and DUNE, will open a new era in studies of supernova and presupernova neutrinos [1, 2], which can give important information for the explosion mechanism of collapse-driven supernovae. This work is dedicated to the neutrino evolution in the astrophysical environment, taking into account the collective neutrino oscillations between different helical states engendered by interaction of the neutrino magnetic moment with a magnetic field, as well as the interaction with matter in the case when there is a transversal matter current or matter polarization. By using the numerical simulation method, we show that distinct spin-flavor spectral splits do exist in the supernova neutrino flavor evolution when there is non-trivial transversally moving matter.

40th International Conference on High Energy physics - ICHEP2020

July 28 - August 6, 2020

Prague, Czech Republic (virtual meeting)

\footnotetext{
${ }^{*}$ Speaker
} 


\section{Neutrino evolution in astrophysical environment}

Consider two flavor neutrinos with two possible helicities $v_{f}=\left(v_{e}^{-}, v_{x}^{-}, v_{e}^{+}, v_{x}^{+}\right)$, where $v_{x}$ stands for $v_{\mu}$ or $v_{\tau}$. Then the neutrino system is described by density matrix

$$
\rho=\left(\begin{array}{cc}
\rho_{\nu} & X \\
X^{\dagger} & \rho_{\bar{v}}
\end{array}\right) \quad \text { and } \quad \rho^{c}=\left(\begin{array}{cc}
\rho_{\bar{v}} & X^{*} \\
X^{T} & \rho_{\nu}
\end{array}\right),
$$

where $\rho$ and $\rho_{\bar{\nu}}$ are the usual $2 \times 2$ flavour density matrices. In case of Dirac neutrinos the matrices describe active and sterile neutrinos, respectively, while in case of Majorana neutrinos $\rho_{\bar{v}}$ describes antineutrinos. External magnetic field and transversally moving matter leads to coupling between $\rho$ and $\rho_{\bar{\nu}}$ (see [3] and references therein) and, therefore, we should also consider non-diagonal matrices $X$

$$
X=\left(\begin{array}{ll}
\rho_{v_{e} \bar{v}_{e}} & \rho_{v_{e} \bar{v}_{x}} \\
\rho_{v_{x} \bar{v}_{e}} & \rho_{v_{x} \bar{v}_{x}}
\end{array}\right) .
$$

The evolution of $\rho$ is governed by Liouville-von Neumann equation of motion

$$
i \frac{d \rho}{d t}=[H, \rho],
$$

where $H$ is a Hamiltonian that describes external environment, which consists of four parts, $H=$ $H_{\text {vac }}+H_{\text {mat }}+H_{v}+H_{\mathrm{B}}+H_{v \nu}$, where $H_{\text {vac }}$ is a vacuum, Hamiltonian, $H_{\text {mat }}$ is a matter potential for electrons and neutrons moving in arbitrary directions, $H_{\mathrm{B}}$ is the Hamiltonians that account for the magnetic field. The neutrino-neutrino interaction potential, $H_{v v}$, is

$$
H_{v v}=\sqrt{2} G_{F} n_{v} \int d E\left[G^{\dagger}\left(\rho(E)-\rho(E)^{c *}\right) G+\frac{1}{2} G^{\dagger}\left[\left(\rho(E)-\rho(E)^{c *}\right) G\right]\right],
$$

with $G_{F}$ being the Fermi coupling constant and $n_{v}$ being neutrino density profile, and $G=$ $\operatorname{Diag}\{+1,+1,-1,-1\}$ is the dimensionless matrix. The explicit forms of Hamiltonians depend on the neutrino nature. Now we consider Majorana neutrinos for illustration.

For the Majorana neutrinos, the diagonal magnetic moments are zero in both flavour and mass basis (see, for instance, [4]). Therefore, it is convenient to introduce the magnetic moment matrix as

$$
\mu_{\alpha \beta}=\left(\begin{array}{cc}
0 & i \mu \\
-i \mu & 0
\end{array}\right)
$$

Then the Hamiltonian for Majorana neutrinos in the magnetic field is expressed as

$$
H_{\mathrm{B}}^{\mathrm{M}}=i \mu \cos 2 \theta\left(\begin{array}{cccc}
0 & \frac{1}{\gamma_{12}} B_{\|} & 0 & -B_{\perp} e^{i \phi} \\
-\frac{1}{\gamma_{12}} B_{\|} & 0 & B_{\perp} e^{i \phi} & 0 \\
0 & -B_{\perp} e^{-i \phi} & 0 & -\frac{1}{\gamma_{12}} B_{\|} \\
B_{\perp} e^{-i \phi} & 0 & \frac{1}{\gamma_{12}} B_{\|} & 0
\end{array}\right) .
$$

The effect of transversally moving matter can be taking into account by shifting of non-diagonal neutrino magnetic moments and by introducing effective diagonal magnetic moments. The parallel moving matter can be expressed as an effective mass at rest. Therefore, it useful to introduce the effective Hamiltonian accounting for external environment that Hamiltonian can be used for the analytical analysis of collective neutrino oscillations. The full and comprehensive analyses will be reported in the future publication [5]. 


\section{Numerical solutions for collective oscillations}

We present our numerical calculations for some specific cases in order to highlight the interplay of neutrino flavor, spin and collective oscillations. In fig. 1, we illustrate the initial (dashed, $r=50$ $\mathrm{km}$ ) and final (solid, $r=200 \mathrm{~km}$ ) supernova neutrino spectra for different neutrino species as a function of the neutrino energy for the normal (left) and inverted (right) mass ordering. The average energies of $v_{e}, \bar{v}_{e}$ and $v_{x}$ are taken as $10 \mathrm{MeV}, 15 \mathrm{MeV}$, and $24 \mathrm{MeV}$, respectively. Note that the bulb model for supernova neutrino emission and single-angle approximation have been used for the numerical calculation, and A, B, C, D in the left panel are cross points for illustration. The transverse matter effects are taken from Ref. [3] in the case of Majorana neutrinos.

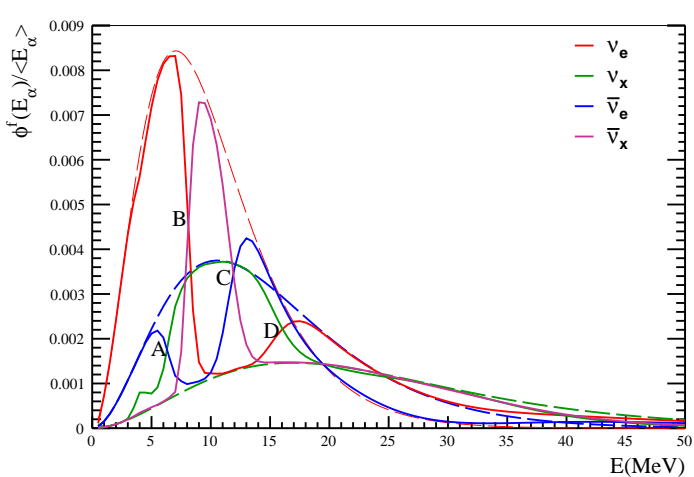

a)

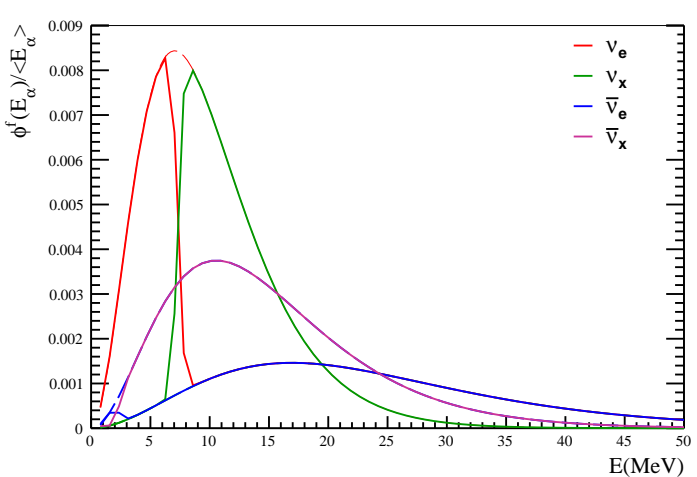

b)

Figure 1: Initial (dashed, $r=50 \mathrm{~km}$ ) and final (solid, $r=200 \mathrm{~km}$ ) supernova neutrino spectra for different neutrino species as a function of the neutrino energy for the normal (left) and inverted (right) mass ordering. The average energies of $v_{e}, \bar{v}_{e}$ and $v_{x}$ are taken as $10 \mathrm{MeV}, 15 \mathrm{MeV}$, and $24 \mathrm{MeV}$, respectively. Note that the bulb model for supernova neutrino emission and single-angle approximation have been used for the numerical calculation, and A, B, C, D in the left panel are cross points for illustration. The transverse matter effects are taken from Ref. [3] in the case of Majorana neutrinos.

This work was supported by the joint project of the National Natural Science Foundation of China (No. 1191101364), and the Russian Foundation for Basic Research (No. 20-52-53022GFEN-a), by the National Natural Science Foundation of China under Grant No. 11835013, and No. 12075255, by Beijing Natural Science Foundation under Grant No. 1192019.

\section{References}

[1] T. Totani, K. Sato, H. Dalhed and J. Wilson, Astrophys. J. 496 (1998), 216-225.

[2] H. L. Li, Y. F. Li, L. J. Wen and S. Zhou, JCAP 05 (2020), 049.

[3] P. Pustoshny and A. Studenikin, Phys. Rev. D 98 (2018) 113009.

[4] C.Giunti, A.Studenikin, Rev. Mod. Phys. 87 (2015) 531.

[5] Z. Yuan, K. Kouzakov, Y.-F. Li, V. Shakhov, K. Stankevich, A. Studenikin, to be published. 